\title{
HVEGF26-104/RFASE Peptide Vaccine
}

National Cancer Institute

\section{Source}

National Cancer Institute. hVEGF26-104/RFASE Peptide Vaccine. NCI Thesaurus. Code C118364.

A therapeutic cancer vaccine containing a truncated, synthetic peptide mimic of the human angiog enic activator vascular endothelial growth factor (VEGF), consisting of 79 amino acids (amino acids 26-104 of VEGF), and emulsified in the immunoadjuvant RFASE, with potential immunostimulatory and antitumor activities. Upon intramuscular vaccination, the hVEGF26-104 moiety of hVEGF26-104/RFASE acts as an antigen and induces an immune response against VEGF, which results in anti-VEGF antibody binding to and neutralization of endogenous VEGF. This prevents the binding of endogenous VEGF to the VEGF receptor (VEGFR) and blocks VEGFR-mediated endothelial cell signaling, resulting in an inhibition of both angiogenesis and tumor cell proliferation. VEGF plays a key role in angiogenesis, tumor cell proliferation and invasion. RFASE, which belongs to the group of sulpholipopolysaccharides (SLPS), is a synthetic polysaccharide covalently coupled to lipid groups and sulphate groups, and is able to induce a strong humoral immune response upon antigen administration. 